\title{
Plasmonic nano-slits assisted polarization selective detour phase meta-hologram
}

\author{
Changjun Min ${ }^{1, \#, *}$, Jinpeng Liu, ${ }^{2, \#}$, Ting Lei ${ }^{1, \#}$, Guangyuan $\mathrm{Si}^{3}$, Zhenwei $\mathrm{Xie}^{1}$, Jiao $\mathrm{Lin}^{1,3,4}$, Luping $\mathrm{Du}^{1}$, and \\ Xiaocong Yuan ${ }^{1, *}$ \\ ${ }^{1}$ Nanophotonics Research Centre, Shenzhen University \& Key Laboratory of Optoelectronic Devices \\ and Systems of Ministry of Education and Guangdong Province, College of Optoelectronic \\ Engineering, Shenzhen University, 518060, China \\ ${ }^{2}$ Institute of Modern Optics, Nankai University, Tianjin 300071, China \\ ${ }^{3}$ School of Engineering, RMIT University, Melbourne, VIC 3001, Australia \\ ${ }^{4}$ School of Physics, University of Melbourne, VIC 3010, Australia \\ *Corresponding Author: Changjun Min, Xiaocong Yuan \\ E-mail: cjmin@szu.edu.cn, xcyuan@szu.edu.cn \\ \#These authors contributed equally to this paper
}

Traditional detour-phase hologram is a powerful optical device for manipulating phase and amplitude of light, but it is usually not sensitive to the polarization of light. By introducing the light-metasurface interaction mechanism to the traditional detour phase hologram, we design a novel plasmonic nanoslits assisted polarization selective detour phase meta-hologram, which has attractive advantages of polarization multiplexing ability, broadband response, and ultra-compact size. The meta-hologram relies on the dislocations of plasmonic slits to achieve arbitrary phase distributions, showing strong polarization selectivity to incident light due to the plasmonic response of deep-subwavelength slits. To verify its polarization sensitive and broadband responses, we experimentally demonstrate two holographic patterns of an optical vortex and an Airy beam at p- and s-polarized light with wavelengths of $532 \mathrm{~nm}, 633 \mathrm{~nm}$ and $780 \mathrm{~nm}$, respectively. Furthermore, we realize an application example of the meta-hologram as a polarization multiplexed photonic device for multi-channel optical angular momentum (OAM) generation and detection. Such meta-holograms could find widespread applications in photonics, such as chip-level beam shaping and high-capacity OAM communication.

Traditional detour phase hologram is not sensitive to polarization of light. Here, a novel detour phase meta-hologram is proposed assisted by plasmonic nano-slits, which shows polarization multiplexing ability, broadband response, and ultra-compact size. The meta-hologram could generate two different holographic patterns at $\mathrm{p}$ - and s-polarized lights, respectively, in visible wavelengths. It is also demonstrated as a polarization multiplexed photonic device for multi-channel optical angular momentum generation and detection

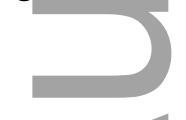

This is the author manuscript accepted for publication and has undergone full peer review but has not been through the copyediting, typesetting, pagination and proofreading process, which may lead to differences between this version and the Version of Record. Please cite this article as doi: 10.1002/lpor.201600101.

This article is protected by copyright. All rights reserved. 


\section{WILEY-VCH}

Keywords: metasurface, detour phase, polarization multiplexing, OAM generation and detection

\section{Introduction}

The concept of detour-phase hologram, introduced by Brown and Lohmann in 1966 [1, 2], has widely been used in computer-generated holograms for applications of beam shaping, three-dimensional display, optical signal processing, and others [3]. Traditional detour-phase hologram usually consists of transmission apertures on an opaque surface, allowing the binary transmittance of only zero or one across the surface. Although such a hologram can modulate phase and amplitude of light based on the apertures' positions and sizes, however, it is not sensitive to the polarization of light, and thus it cannot shape the polarization status of a light beam or work as polarization-multiplexing devices.

In recent years, metasurface [4] with subwavelength sized features is proposed to significantly improve the performance of traditional optical devices by engineering the interaction between incident light and subwavelength optical scatters (or optical antennas) [5-7], such as metallic nanorods [8-10] and V-shaped metallic nano-antennas [4]. Due to its very attractive features of micron-scale size, nanoscale thickness, broadband response from visible to infrared frequency, and multi-functions (manipulating phase, amplitude and polarization), metasurface has shown great application prospects in many optical fields, such as aberration-free lenses and axicons [11], micrometer-scale quarter wavelength plate [12], photonic spin hall effect [13], optical vortex generations [14-16], detection of multiple optical angular momentum (OAM) [17], and 3dimentional holograms [18]. Thus, metasurface could be a very promising approach to improve the performance of traditional detour-phase hologram, especially the polarization response property. Lin et al. first demonstrated a metasurface-based detour-phase hologram [19], however, in this design the detour-phase hologram was still not sensitive to the polarization, so another polarizationsensitive metallic nanostructure was combined to the hologram, which requires two independent complex structures to control the phase and polarization, respectively.

In this paper, through revisiting the concept of traditional detour phase hologram, we design and demonstrate a new type of polarization-sensitive detour phase hologram based on a metasurface composed of plasmonic nano-slits on a metal film. These nano-slits have uniform shape and orientation but different positions in a period of metasurface to obtain arbitrary phase distribution

This article is protected by copyright. All rights reserved. 


\section{WILEY-VCH}

of transmitted beam, and thus form a new type of metasurface hologram (meta-hologram). Besides the phase modulation, such a deep-subwavelength slit on metal film also has a strong polarization sensitivity, due to the fact that only p-polarized incident light can excite surface plasmon polaritons (SPP) to transmit through the nano-slits, while s-polarized incident light is almost reflected by the metallic surface. By combining different responses to $p$ - and s-polarization, we finally obtain a polarization multiplexed meta-hologram. To verify the capability of such meta-holograms, we demonstrate the generation of an $\mathrm{OV}$ and an Airy beam at $\mathrm{p}$ - and s-polarized incidences, respectively, by both experiments and finite difference time domain (FDTD) calculations. In the experiments, we choose three different wavelengths of $532 \mathrm{~nm}, 633 \mathrm{~nm}$, and $780 \mathrm{~nm}$ to confirm its broadband response feature. In addition, we show the generation of two $3 \times 3$ OAM arrays for $p$ - and s-polarizations and the detection of incident OAMs by a designed meta-hologram, to further demonstrate its great potential as photonic devices for OAM generation, detection and modulation. We believe that the detour phase meta-hologram benefits from the advantages of polarization multiplexing ability, ultra-compact size, and broadband response, therefore has promising applications in a wide field, such as chip-level beam shaping and high-capacity OAM communication.

\section{Principle and structure design}

The principle of detour phase in the meta-hologram is shown in Figure 1(a). When a light beam is incident into two adjacent subwavelength sized slits, it will be diffracted at each slit and produce a second wave of equal phase, like two parallel line sources. When receiving the diffraction wave at a fixed angle $\vartheta$, the two wavefronts have an optical path difference $\Delta L=D \sin \vartheta$, which gives a corresponding phase difference $\Delta \varphi=2 \pi D \sin \vartheta / \lambda$ depending on the distance $D$ between adjacent slits. Therefore, we can control the phase difference by adjusting the distance $D$. When $D=n \lambda / \sin \vartheta(n=1$, $2 \ldots)$, the phase difference $\Delta \varphi$ generated by the two slits is equivalent to zero; in other cases the phase difference can be selected from 0 to $2 \pi$ according to different values of $D$. Furthermore, we extend the two slits to a periodic slit array and choose the period length $P=D=\lambda / \sin \vartheta$ at $n=1$, then we can determine the phase difference of each slit from 0 to $2 \pi$ through adjusting the slit position in each period. To form a hologram with two-dimensional phase distribution $\varphi_{(i, j)}$, the slit position in each period is determined by a profile of $D_{(i, j)}=P \varphi_{(i, j)} / 2 \pi$, where $D_{(i, j)}$ is the location shift of the slit $(i, j)$ to the center of its period, $P=\lambda / \sin \vartheta$ is the period length, and $\varphi_{(i, j)}$ is in the range of $-\pi \sim \pi$. Finally, a

This article is protected by copyright. All rights reserved. 


\section{WILEY-VCH}

desired phase distribution is obtained by a two-dimensional slit array with different slit location shift $D_{(i, j)}$. The generated holographic pattern is received at the angle $\vartheta=\sin ^{-1}(\lambda / P)$ which can also be tuned by the ratio of $\lambda / P$.

It is noted that compared to a conventional detour phase hologram, here the meta-hologram composed of plasmonic nano-slits shows a strong polarization sensitivity, because the deepsubwavelength width of slit only allows the evanescent SPP propagating mode to pass through, while all other propagating waveguide modes of slit are prohibited, similar to the case of well-known metal/insulator/metal (MIM) waveguide [20]. Thus, only p-polarized light that is able to excite SPP could transmit through the slits and form desired meta-hologram, while s-polarized light that cannot excite SPP is almost reflected by the opaque metal film. Such polarization sensitivity provides an important function of polarization multiplexing for the meta-hologram, which greatly increases the information contained in the meta-hologram without enlarging the device size.

The detailed design procedure of the meta-hologram is introduced in Figure 1(b). We first design two conventional phase holograms for generating an OV with topological charge $l=10$ and an Airy beam, respectively, and then convert the phase holograms to two meta-holograms composed of horizontal and vertical nano-slit arrays, respectively, according to the detour phase principle. Finally, we combine the two orthogonal nano-slit arrays into one meta-hologram with cross-shaped slits. To demonstrate the polarization multiplexing property of the synthetic plasmonic meta-hologram with cross-shaped slits, we show both near-field and far-filed distributions of transmitted light intensity in Figure 1(b) illuminated by two orthogonal linear-polarized lights along horizontal and vertical directions, respectively, calculated using the FDTD method. It is observed that in the near field the vertical linear-polarized light only excites SPP with horizontal slits, while the horizontal linearpolarized light only excites SPP with vertical slits, thus the two corresponding patterns of OV and Airy beam are generated in the far field at the orthogonal polarizations, respectively.

Figure $1(\mathrm{c})$ shows the schematic of the polarization multiplexed meta-hologram illuminated by a $45^{\circ}$ linear-polarized light. The meta-hologram was etched in a $200 \mathrm{~nm}$-thick gold film on glass substrate, which consists of totally $40 \times 40$ periods with each period length of $1 \mu \mathrm{m}$. The length of slit in each period is fixed as $1 \mu \mathrm{m}$, same as the period length in order to increase the light transmission.

This article is protected by copyright. All rights reserved. 


\section{WILEY-VCH}

In this case, due to the $45^{\circ}$ linear polarization of incident light, SPP are excited by both horizontal and vertical slits at the same time, hence the holographic patterns in horizontal and vertical directions both appear in the far field. It is noted that the desired OV and Airy patterns appear at two symmetrical diffraction angles of $\pm \vartheta$, similar to the \pm 1 diffraction orders of optical grating, and the angle $\vartheta$ could be modulated by the period length and the wavelength according to the equation $P=\lambda / \sin \vartheta$.

\section{Theoretical analysis}

To investigate the influences of parameters of the meta-hologram to its performance, in Figure $\mathbf{2}$ we show the FDTD calculated results of transmittance, efficiency and polarization extinction ratio as functions of the thickness of gold film, slits width, and the incident wavelength. Here the transmittance is defined as the ratio of total transmitted energy to total incident energy, the efficiency represents the energy of desired pattern at $\pm \vartheta$ diffraction angles over the total incident energy, and the polarization extinction ratio is the ratio of efficiency at p-polarized incidence to the one at s-polarized incidence. The larger polarization extinction ratio is corresponding to better polarization selectivity of the device, and based on our study the polarization selectivity phenomenon is quite obvious when the polarization extinction ratio is larger than 10.

In Figure 2(a) we consider the performance of the meta-hologram at different wavelength of incident light. The results verify that the meta-hologram has a broadband spectrum response, especially in the range of $500 \mathrm{~nm}-900 \mathrm{~nm}$ a relatively good far field pattern can be observed. We can see that the highest efficiency of $\sim 9 \%$ is obtained in $600 \mathrm{~nm}-700 \mathrm{~nm}$ range, while the polarization extinction ratio is also relatively high as $\sim 100$. Different to previous metasurfaces whose broadband feature usually origins from the overlap of several resonance modes of nano-antennas, here the working wavelength for detour phase obeys the equation $P=\lambda / \operatorname{sin\vartheta }$, hence different wavelengths could be used to generate same holographic pattern while only the observed angle (diffraction angle $\vartheta$ ) changes at the fixed period length $P$. Figure $2(d)$ gives the relationship between the diffraction angle $\theta$ and the period length $P$ at $532 \mathrm{~nm}, 633 \mathrm{~nm}$, and $780 \mathrm{~nm}$ wavelengths, which shows that the working wavelength range of meta-hologram is mainly limited by the maximum diffraction angle $\vartheta$,

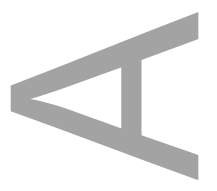

This article is protected by copyright. All rights reserved. 


\section{WILEY-VCH}

but can be further increased with a larger period length $P$. In addition, the working wavelength range is also affected by the loss of metal, which results to low transmittance at small wavelength.

In Figs. 2 (b) and 2(c) we study the effects of slit width and gold film thickness at the fixed wavelength of $633 \mathrm{~nm}$, respectively. When the slit width increases, the transmittance and the efficiency can be greatly improved as more incident light could pass through, but the polarization extinction ratio is exponentially reduced due to the fact that larger width also allows s-polarized light to directly pass through. And when the gold film thickness increases, the polarization extinction ratio becomes higher because of the increased loss of metal to s-polarized light, while the efficiency and the transmittance reach a peak value at about 150nm thickness, the reason is that the excited SPP in the slit achieves a cavity resonance mode at that thickness and thus enhances the transmittance. Based on above results, we find that there is usually a trade-off between the efficiency and the polarization extinction ratio when the structure parameters change, so it should be careful to choose the parameters for various requirements.

\section{Experimental verification}

In order to experimentally verify the effectiveness of our designed meta-hologram, we used the focused ion beam (FIB) method to fabricate $40 \times 40 \mu \mathrm{m}^{2}$ meta-holograms, and built an experimental system as shown in Figure 3(a). In the system, three incident wavelengths of 532nm, $633 \mathrm{~nm}$ and $780 \mathrm{~nm}$ were chosen to demonstrate its broadband feature. The incident laser beam was first collimated and expanded by two lens, and then converted to linear polarization by a polarizer and its polarization direction was adjusted by rotating a half wave plate. After, we used a $4 \times$ objective lens to focus the beam onto the meta-hologram. Finally, to cover the large diffraction angles at the three wavelengths, we chose a $100 \times$ objective lens to receive all the diffraction patterns and recorded them by a CCD.

Figure 3(b) gives the scanning electron microscope (SEM) photo of the fabricated meta-hologram, and its corresponding theoretically predicted diffraction pattern of an Airy beam and an OV at the $45^{\circ}$ linear-polarized incidence is shown in Figure 3(c). Here we chose $50 \mathrm{~nm}$ slit width and $200 \mathrm{~nm}$

This article is protected by copyright. All rights reserved. 


\section{WILEY-VCH}

thickness of gold film in order to obtain a large polarization extinction ratio and clearly show the polarization multiplexing function. In Figs. 3(d)-3(f) we compared the theoretical and experimental results of the OV and airy beam polarization multiplexing hologram, with incident lights of $45^{\circ}, 0^{\circ}$ and $90^{\circ}$ linear polarizations and $532 \mathrm{~nm}, 633 \mathrm{~nm}$ and $780 \mathrm{~nm}$ wavelengths, respectively. It is obvious that for different wavelengths, the meta-hologram always shows strong polarization selectivity in experiment. In the case of $0^{\circ}$ or $90^{\circ}$ linearly polarized incident light, only the slits perpendicular to the polarization direction can excite SPP and work as a hologram, thus only the Airy beam or the OV could be observed in one direction. They can be both observed with a $45^{\circ}$ linear polarized incident light which can be divided into $0^{\circ}$ and $90^{\circ}$ linear polarizations. It is noted that when the incident wavelength $\lambda$ increases, the whole holographic pattern becomes larger due to the increasing diffraction angle $\vartheta=\sin ^{-1}(\lambda / P)$ at fixed period length $P$, and the measured polarization extinction ratios for wavelengths of $532 \mathrm{~nm}, 633 \mathrm{~nm}$ and $780 \mathrm{~nm}$ are $231.51,599.49$, and 873.57 , respectively, which are quite large and consistent with the increasing trend shown in shown in Figure 2(a). In experimental results, the shape of OV somehow looks like square instead of circle, owing to the Fraunhofer diffraction effect of the square entrance pupil of the meta-hologram sample illuminated by a larger size beam.

\section{OAM generation and detection}

To further demonstrate the great potential of our designed meta-holograms as photonic devices, we consider using it as a polarization multiplexed devices for OAM generation and detection. As a new degree of freedom for information encoding and decoding, in recent years OAM has been proved to have significant potential in free-space optical communication system to realize high-capacity and multi-channel communications [21-27]. And the practical applications of OAM require photonic integrated devices and circuits for miniaturization, improved performance, and enhanced functionality [23]. Consequently, we designed and fabricated a polarization multiplexed metahologram as an OAM generation and detection device, as shown in Figure 4.

Figure 4(a) presents the SEM photo of the fabricated meta-hologram designed by the detour phase distribution of a 2-dimentinal Dammann vortex grating [28], and figure 4(b) gives the

This article is protected by copyright. All rights reserved. 


\section{WILEY-VCH}

corresponding predicted diffraction pattern of a $3 \times 3$ OAM array at the $45^{\circ}$ linear-polarized incidence. The experimental results of the $3 \times 3$ OAM array generations at $532 \mathrm{~nm}$ and $633 \mathrm{~nm}$ wavelengths and $45^{\circ}, 0^{\circ}$ and $90^{\circ}$ linear polarizations are compared in Figs. $4(\mathrm{c})$ and $4(\mathrm{~d})$. Here we do not show the results at $780 \mathrm{~nm}$ because its diffraction angle is out of the range of the objective lens. When illuminated by a plane wave with topological charges $l=0$, the generated $3 \times 3$ OAM array contains 9 OAMs with topological charges $l=-4^{\sim}+4$, and each OAM can work as a channel for data communication, thus the meta-hologram could provide 9 channels with OAM multiplexing, and totally 18 channels with multiplexing of both OAM and orthogonal $0^{\circ} / 90^{\circ}$ linear polarizations as all the channels are spatially separated. Besides generation of OAM array, in Figs. 4(c) and 4(d) we show that the meta-hologram can also be used to detect the OAM and linear polarization state of an incident OV beam. When an OV beam with topological charge $+\mathrm{l}$ is incident onto the meta-hologram, it will be restored to a bright point at the location corresponding to topological charge -l of the farfield OV array, while other locations still hold the ring of OV with a dark center. For example, in Figure 4 (c) we chose an OV with $l=+1$ incident onto the meta-hologram with $0^{\circ}$ or $90^{\circ}$ linear polarization, and detected a bright point at the location of the $l=-1$ in corresponding horizontal or vertical direction. Thus, the meta-hologram is able to generate and detect OAMs, and increase the processing information capacity through multiplexing of both OAMs and polarizations, which proves it a promising photonic device for high-capacity and multi-channel OAM communications.

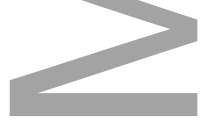

\section{Conclusion and outlook}

In summary, to improve the performance of traditional detour phase hologram, we proposed and experimentally demonstrated a novel plasmonic nano-slit assisted polarization selective detour phase meta-hologram, showing advantages of polarization multiplexing ability, ultra-compact size, and broadband response. Based on the superposition of horizontal and vertical nano-slits corresponding with two phase holograms, we achieved the polarization multiplexing feature for two orthogonal linear polarizations, and observed two different holographic patterns in the far field. We investigated the effects of incident wavelength, slit width, and gold film thickness on the performances of meta-hologram such as transmittance, efficiency, and polarization extinction ratio.

This article is protected by copyright. All rights reserved. 


\section{WILEY-VCH}

The meta-hologram shows a broadband response of $500 \mathrm{~nm}-900 \mathrm{~nm}$ and tunable diffraction angles. We further fabricated a few samples of the meta-hologram by FIB technique, and experimentally verified its broadband response and polarization multiplexing function. Finally, we demonstrated an application example of the meta-hologram as a polarization multiplexed photonic device for OAM generation and detection, which could increase the channels for data communication and has great potential in high-capacity multi-channel OAM communications. Based on the attractive features of the plasmonic nano-slit assisted detour phase meta-hologram, we believe such a novel device could find widespread applications in photonics.

As final remarks, the meta-hologram has the potential of further improving the performance. Similar to other transmission plasmonic metasurfaces $[4,8,29]$, although the efficiency of metahologram is not high, it could be enhanced by changing the transmission structure to the reflection type which has been proved a very high efficiency for plasmonic metasurface [30]. It is also very possible to achieve high-efficiency detour phase meta-hologram by all-dielectric metasurface due to its low-loss feature [31, 32], and very recently an example was reported by Capasso's group [33]. Besides shaping the phase distribution, the amplitude of light can also be controlled by changing the slit width or the number of slits in a period of the meta-hologram. Further, in addition to the linear polarization, the nano-slit structure can be changed to other structures such as $\lambda$-shaped aperture $[34,35]$, which is possible to achieve the chiral polarization multiplexing for hologram.

\section{Acknowledgements}

This work was supported by the National Natural Science Foundation of China under Grant Nos.61138003, 61427819, 61422506, 61490712, and 61405121; National Key Basic Research Program of China (973) under grant No.2015CB352004; National Key Research and Development Program of China under grant No.2016YFC0102401; Science and Technology Innovation Commission of Shenzhen under grant Nos.KQCS2015032416183980, KQCS2015032416183981, JCYJ20140418091413543, and the start-up funding at Shenzhen University. X.Y. appreciates the support given by the leading talents of Guangdong province program No. 00201505.

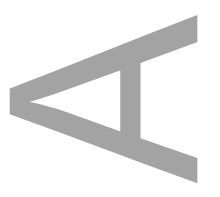

This article is protected by copyright. All rights reserved. 


\section{WILEY-VCH}

\section{References}

[1] B. R. Brown and A. W. Lohmann, Appl. Opt. 5, 967-969, (1966).

[2] A. W. Lohmann and D. P. Paris, Appl. Opt. 6, 1739-1748, (1967).

[3] T. C. Poon, Digital holography and three-dimensional display (Springer, 2006).

[4] N. Yu, P. Genevet, M. A. Kats, F. Aieta, J. P.Tetienne, F. Capasso, and Z. Gaburro, Science 334, 333-337, (2011).

[5] A. V. Kildishev, A. Boltasseva, and V. M. Shalaev, Science 339, 1232009, (2013).

[6] N. Yu and F. Capasso, Nat. Mater. 13, 139-150, (2014).

[7] J. Lin, Q. Wang, G. H. Yuan, L. P. Du, S. S. Kou, and X-C. Yuan, Sci. Rep. 5, 10529, (2015).

[8] L. Huang, Chen, H. Mühlenbernd, G. Li, B. Bai, Q. Tan, G. Jin, T. Zentgraf, S. Zhang, Nano Lett. 12, 5750-5755, (2012).

[9] D. Wen, F. Yue, G. Li, G. Zheng, K. Chan, S. Chen, M. Chen, K. F. Li, P. W. H. Wong, K. W. Cheah, E. Y. B. Pun, S. Zhang, and X. Chen, Nat. Commun. 6, 8241, (2015).

[10] Y. Montelongo, J. O. Tenorio-Pearl, W. I. Milne, and T. D. Wilkinson, Nano Lett. 14, 294-298, (2014).

[11] F. Aieta, P. Genevet, M. A. Kats, N. Yu, R. Blanchard, Z. Gaburro, and F. Capasso, Nano Lett. 12, 4932-4936, (2012).

This article is protected by copyright. All rights reserved. 


\section{WILEY-VCH}

[12] N. Yu, F. Aieta, P. Genevet, M. A. Kats, Z. Gaburro, and F. Capasso, Nano Lett. 12, 6328-6333, (2012).

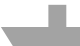

[13] X. Yin, Z. Ye, J. Rho, Y. Wang, and X. Zhang, Science 339, 1405-1407, (2013).

[14] E. Karimi, S. A. Schulz, I. De Leon, H. Qassim, J. Upham, and R. W. Boyd, Light Sci. Appl. 3, e167, (2014).

[15] Y. Yang, W. Wang, P. Moitra, I. I. Kravchenko, D. P. Briggs, and J. Valentine, Nano Lett. 14, 1394-1399, (2014).

[16] K. E. Chong, I. Staude, A. James, J. Dominguez, S. Liu, S. Campione, G. S. Subramania, T. S. Luk, M. Decker, D. N. Neshev, I. Brener, and Y. S. Kivshar, Nano Lett. 15, 5369-5374, (2015).

[17] J. P. Liu, C. J. Min, T. Lei, L. P. Du, Y. S. Yuan, S. B. Wei, Y. P. Wang, and X. C. Yuan, Opt. Express 24, 212-218, (2016).

[18] L. Huang, X. Chen, H. Mühlenbernd, H. Zhang, S. Chen, B. Bai, Q. Tan, G. Jin, K. Cheah, C. Qiu, J. Li, T. Zentgraf, and S. Zhang, Nat. Commun. 4, 2808, (2013).

[19] J. Lin, P. Genevet, M. A. Kats, N. Antoniou, and F. Capasso, Nano Lett. 13, 4269-4274, (2013).

[20] S. A. Maier, Plasmonics: Fundamentals and Applications. (Springer, 2007).

[21] L. Allen, M. W. Beijersbergen, R. J. C. Spreeuw, and J. P. Woerdman, Phys. Rev. A 45, 81858189, (1992).

[22] L. Allen, S. M. Barnett, and M. J. Padgett, Orbital Angular Momentum (Institute of Physics Publishing, 2003).

This article is protected by copyright. All rights reserved. 


\section{WILEY-VCH}

[23] X. Cai, J. Wang, M. J. Strain, B. Johnson-Morris, J. Zhu, M. Sorel, J. L. O’Brien, M. G.

Thompson, and S. Yu, Science 338, 363-366, (2012).

[24] J. Wang, J. Y. Yang, I. M. Fazal, N. Ahmed, Y. Yan, H. Huang, Y. X. Ren, Y. Yue, S. Dolinar, M. Tur, and A. E. Willner, Nat. Photonics 6, 488-496, (2012).

[25] T. Lei, M. Zhang, Y. Li, P. Jia, G. N. Liu, X. Xu, Z. Li, C. Min, J. Lin, C. Yu, H. Niu, and X. C. Yuan, Light Sci. Appl. 4, e257, (2015).

[26] M. J. Strain, X. Cai, J. Wang, J. Zhu, D. B. Phillips, L. Chen, M. Lopez-Garcia, J. L. O’Brien, M. G. Thompson, M. Sorel, and S. Yu, Nat. Commun. 5, 4856, (2014).

[27] S. Yu, Opt. Express 23, 3075-3087, (2015).

[28] N. Zhang, X. C. Yuan, and R. E. Burge, Opt. Lett. 35, 3495-3497, (2010).

[29] L. Huang, H. Mühlenbernd, X. Li, X. Song, B. Bai, Y. Wang, and T. Zentgraf, Adv. Mater. 27, 6444-6449, (2015).

[30] G. Zheng, H. Mühlenbernd, M. Kenney, G. Li, T. Zentgraf, and S. Zhang, Nat. Nanotechnol. 10, 308-312, (2015).

[31] K. E. Chong, L. Wang, I. Staude, A. R. James, J. Dominguez, S. Liu, G. S. Subramania, M. Decker, D. N. Neshev, I. Brener, and Y. S. Kivshar, ACS Photonics 3, 514-519, (2016).

[32] Y.F.Yu, A.Y. Zhu, R. Paniagua-Domínguez, Y. H. Fu, B. Luk'yanchuk, and A. I. Kuznetsov, Laser \& Photon. Rev. 9, 412-418, (2015).

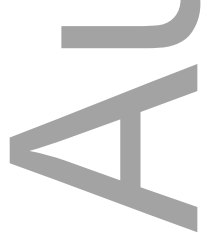

This article is protected by copyright. All rights reserved. 


\section{WILEY-VCH}

[33] M. Khorasaninejad, A. Ambrosio, P. Kanhaiya, and F. Capasso, Science Advances 2, e1501258, (2016).

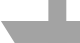

[34] J. Lin, J. P. Balthasar Mueller, Q. Wang, G. H. Yuan, N. Antoniou, X. C. Yuan, and F. Capasso, Science 340, 331-334, (2013).

[35] L. P. Du, S. S. Kou, E. Balaur, J. Cadusch, A. Roberts, B. Abbey, X. C. Yuan, D. Y. Tang, and J. Lin, Nat. Commun. 6, 10051, (2015).

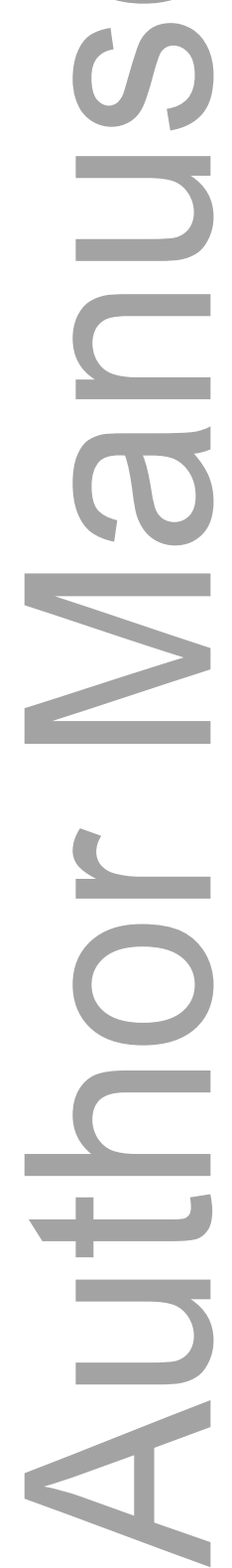

This article is protected by copyright. All rights reserved. 


\section{WILEY-VCH}

Figure 1. Principle of detour phase and polarization multiplexing of meta-hologram. (a) Schematic of detour phase. Each air slit at different locations in a period length P produces different phase $\left(-\pi^{\sim} \pi\right)$. $D$ represents the distance between adjacent slits and $\vartheta$ is the angle of diffraction light. (b) Schematic of the design procedure of the meta-hologram. We successively show the two original phase holograms, the two plasmonic metasurfaces with horizontal and vertical slits for the two phase holograms, the synthetic plasmonic meta-hologram with cross-shaped slits, the near-field distributions of transmitted light intensity for the vertical polarized (P-Pol) or the horizontal polarized (S-Pol) light, and the far-field distributions of an OV with topological charge $l=10$ or an Airy beam for $\mathrm{p}$ - or s-polarized light. (c) Schematic of the meta-hologram illuminated by a $45^{\circ}$ linearpolarized light. The generated holographic patterns of an OV and an Airy beam are produced in the \pm 1 diffraction order at the far field. The cross-shaped silts are etched into a gold film on a glass substrate. The slit length is $1 \mu \mathrm{m}$ same as the period length $P$, and the slit width is defined as $w$.

(a)
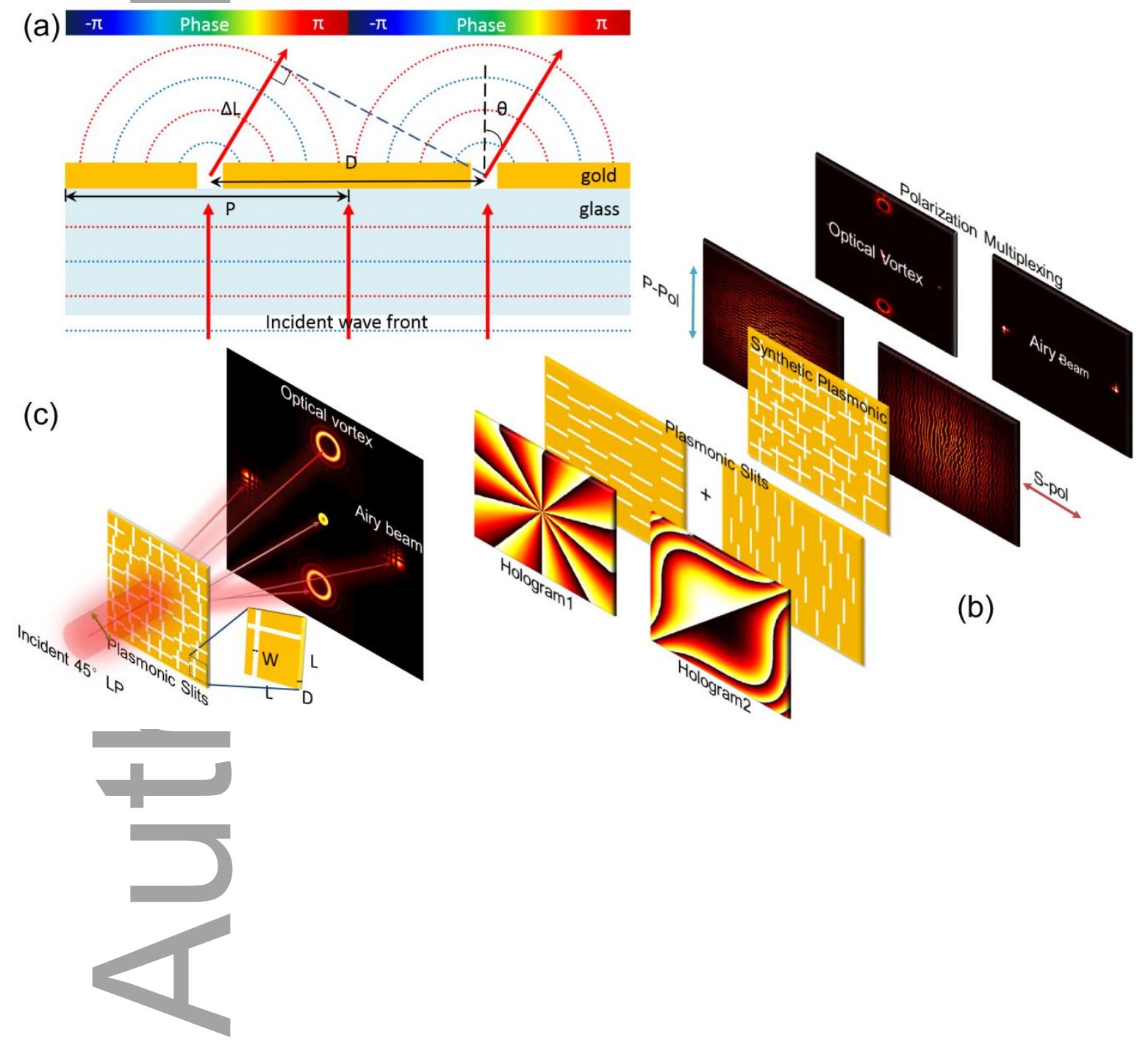

This article is protected by copyright. All rights reserved. 


\section{WILEY-VCH}

Figure 2. Influences of slit parameters and wavelength. (a) - (c) Curves of efficiency (black solid lines), transmittance (black dashed line), polarization extinction ratio (red solid line) as function of wavelength, slit width and gold film thickness calculated by FDTD method. The parameters are chosen as (a) gold film thickness $150 \mathrm{~nm}$, slit width 100nm; (b) gold film thickness $150 \mathrm{~nm}$, wavelength $633 \mathrm{~nm}$; (c) slit width $100 \mathrm{~nm}$, wavelength $633 \mathrm{~nm}$. (d) The relationship between diffraction angle and the period length of the meta-hologram in the wavelengths of $532 \mathrm{~nm}, 633 \mathrm{~nm}$ and $780 \mathrm{~nm}$. The diffraction angle can be adjusted from $20^{\circ}$ to $80^{\circ}$ by changing the period length.
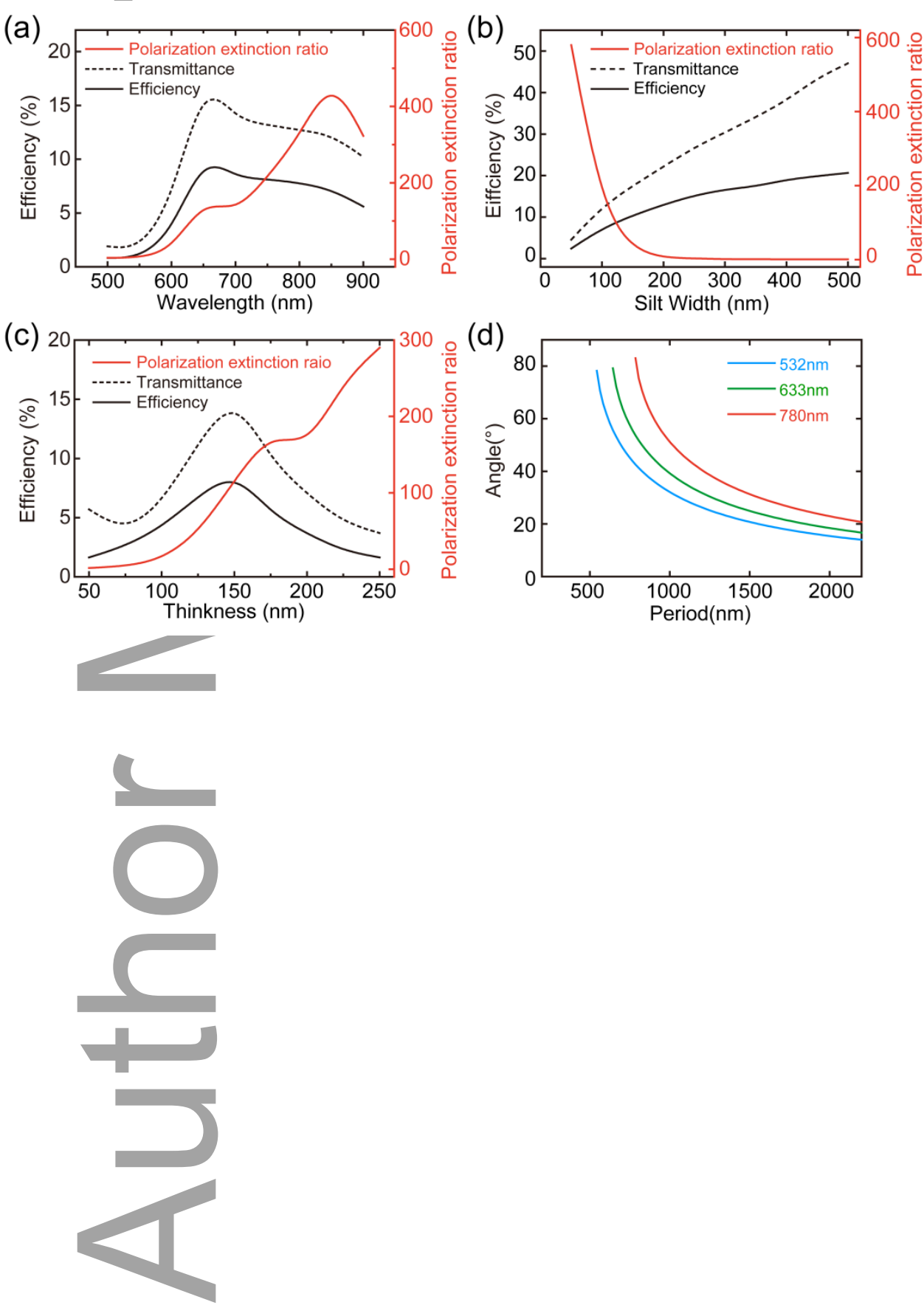

This article is protected by copyright. All rights reserved. 


\section{WILEY-VCH}

Figure 3. Experiment system and results of polarization multiplexing. (a) Experiment system diagram. The light emitted by a laser passes through lens $(L 1, L 2)$ to expansion, and then converted to linearly polarized light by the polarizer (P). Through rotating the half wave plate (HWP), we can adjust the direction of the linear polarization to generate $p$ - or s-polarized light. Then we use a $4 \times$ objective lens (OBJ1) to focus the light onto the sample of meta-hologram and a 100x objective lens to collect diffraction light into the CCD. (b) SEM photo of meta-hologram fabricated by FIB method. The metahologram size is $40 \times 40 \mu \mathrm{m}^{2}$ consisting of $40 \times 40$ nano-slits, which is $1 \mu \mathrm{m}$ length and $50 \mathrm{~nm}$ width etched on $200 \mathrm{~nm}$ thickness gold film. (c) FDTD calculated result of the far-field pattern with $45^{\circ}$ linear-polarized incidence of $633 \mathrm{~nm}$. The white dashed line gives the region of experimental results shown in (d)-(f). (d)-(f) Experimental results of far-field OV and airy beam with $45^{\circ} / 90^{\circ} / 0^{\circ}$ line polarizations at $532 \mathrm{~nm}, 633 \mathrm{~nm}$ and $780 \mathrm{~nm}$, respectively. The white arrow gives the direction of line polarization.

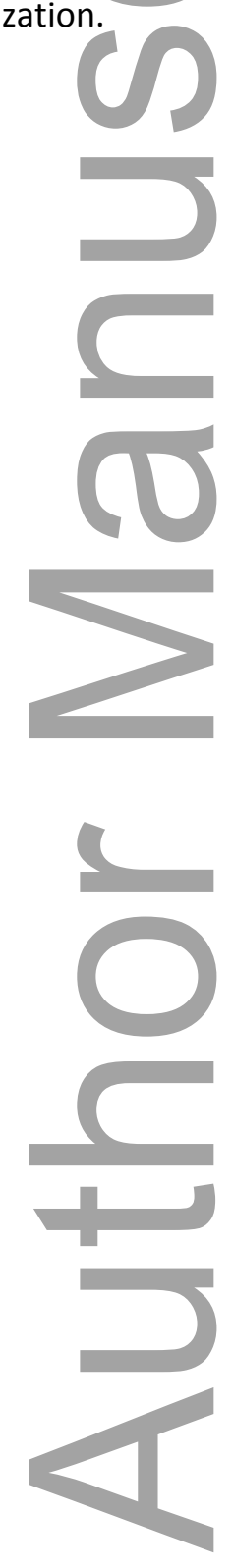

This article is protected by copyright. All rights reserved. 


\section{WILEY-VCH}

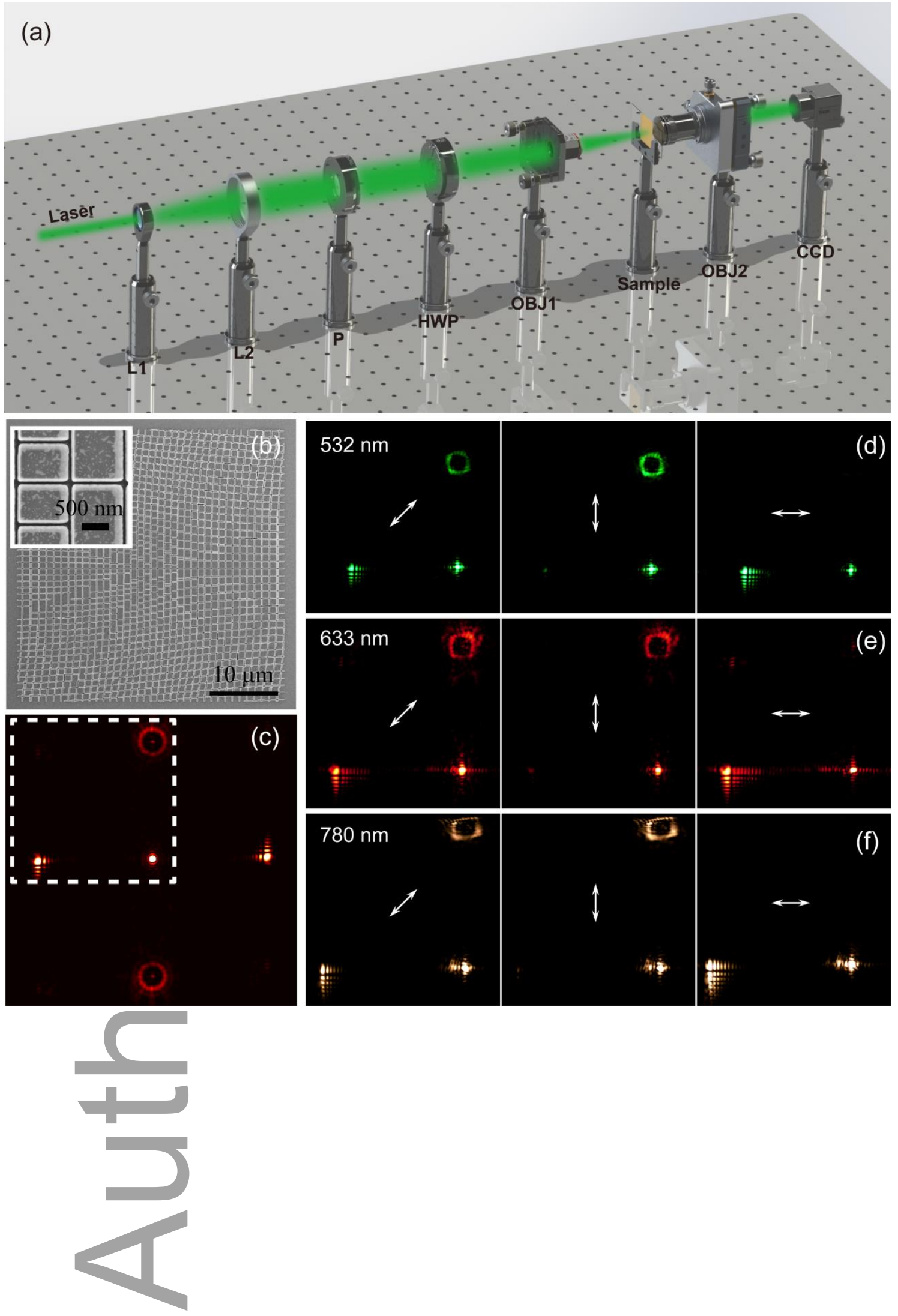

This article is protected by copyright. All rights reserved. 


\section{WILEY-VCH}

Figure 4. Generation and detection of OAMs by the meta-hologram. (a) SEM photo of the sample of meta-hologram. All parameters are same as Figure 3(b). (b) FDTD calculated result of the generation of $3 \times 3$ OAMs array with $45^{\circ}$ linear-polarized incidence of $633 \mathrm{~nm}$. (c)-(d) Experimental results of $3 \times 3$ OAMs array with $45^{\circ} / 90^{\circ} / 0^{\circ}$ line polarizations at $532 \mathrm{~nm}$ and $633 \mathrm{~nm}$, respectively. The white arrow gives the direction of line polarization. The three figures in upper line are corresponding to a plane wave incidence with topological charge $l=0$. The three figures in lower line are corresponding to an OV beam incidence with topological charge $l=1$, and a bright spot is detected at the location of $l=-1$ indicated by a dashed circle.
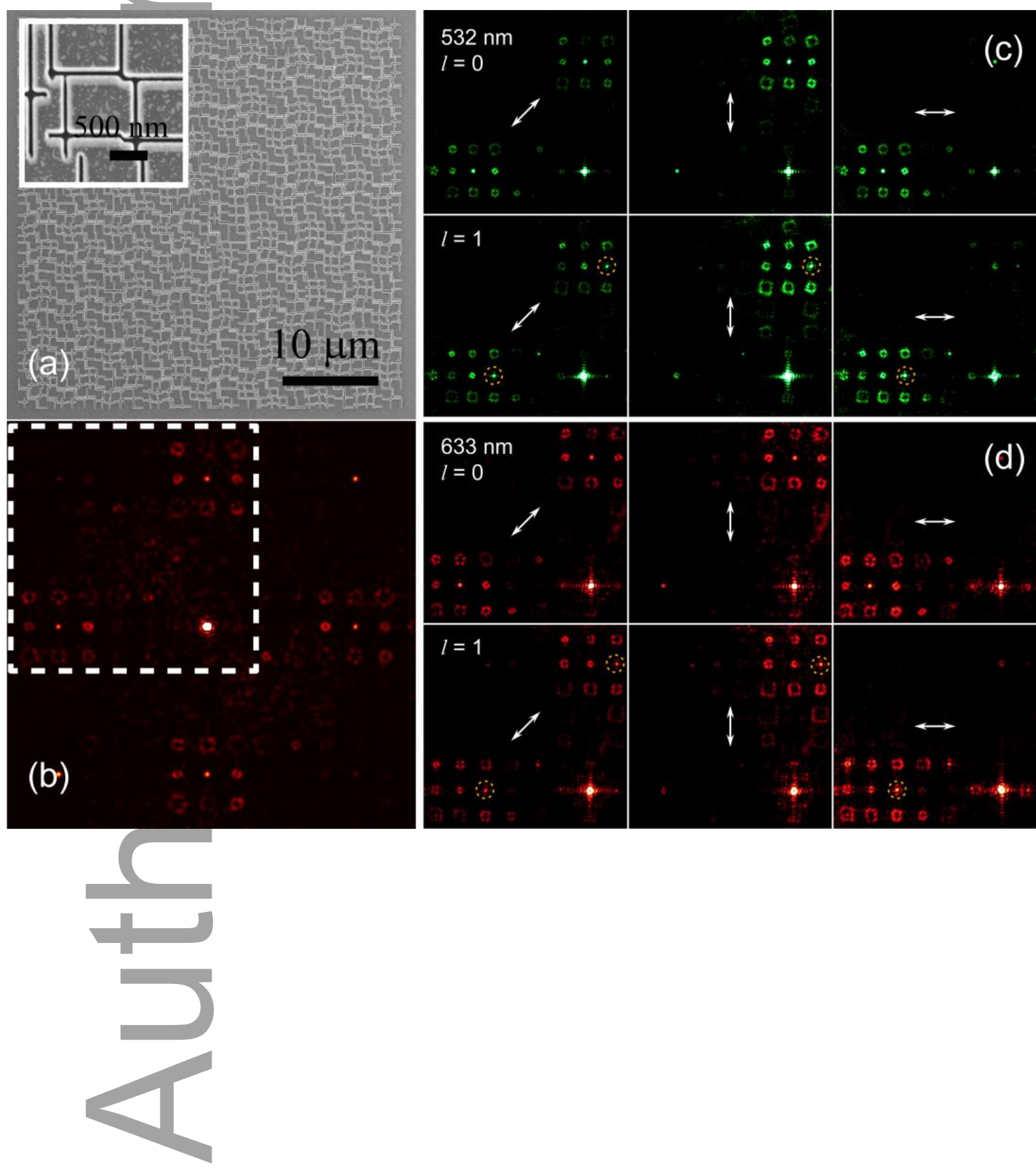

This article is protected by copyright. All rights reserved. 


\section{University Library}

\section{- M M N E R VA A gateway to Melbourne's research publications}

Minerva Access is the Institutional Repository of The University of Melbourne

Author/s:

Min, C;Liu, J;Lei, T;Si, G;Xie, Z;Lin, J;Du, L;Yuan, X

Title:

Plasmonic nano-slits assisted polarization selective detour phase meta-hologram

Date:

2016-11-01

Citation:

Min, C., Liu, J., Lei, T., Si, G., Xie, Z., Lin, J., Du, L. \& Yuan, X. (2016). Plasmonic nano-

slits assisted polarization selective detour phase meta-hologram. LASER \& PHOTONICS REVIEWS, 10 (6), pp.978-985. https://doi.org/10.1002//por.201600101.

Persistent Link:

http://hdl.handle.net/11343/291817 